\title{
Organization of DNA Replication Origin Firing in Xenopus Egg Extracts : The Role of Intra-S Checkpoint
}

\section{Diletta Ciardo}

I2BC: Institut de Biologie Integrative de la Cellule Site d'Orsay

\section{Olivier Haccard}

I2BC: Institut de Biologie Integrative de la Cellule Site d'Orsay

\section{Hemalatha Narassimprakash}

I2BC: Institut de Biologie Integrative de la Cellule Site d'Orsay

\section{Jean-Michel Arbona}

ENS Lyon: Ecole normale superieure de Lyon

\section{Olivier Hyrien}

IBENS: Institut de Biologie de l'Ecole Normale Superieure

\section{Benjamin Audit}

ENS Lyon: Ecole normale superieure de Lyon

\section{Kathrin Marheineke}

I2BC: Institut de Biologie Integrative de la Cellule Site d'Orsay

\section{Arach Goldar ( $\square$ arach.goldar@cea.fr)}

I2BC: Institut de Biologie Integrative de la Cellule Site d'Orsay https://orcid.org/0000-0003-4139-247X

\section{Research}

Keywords: DNA, Xenopus egg, intra-S checkpoint

Posted Date: May 13th, 2021

DOI: https://doi.org/10.21203/rs.3.rs-502134/v1

License: (1) (1) This work is licensed under a Creative Commons Attribution 4.0 International License. Read Full License 


\section{Organization of DNA replication origin firing in Xenopus egg extracts : the role of intra-S checkpoint}

Diletta Ciardo ${ }^{1}$, Olivier Haccard ${ }^{1}$, Hemalatha Narassimprakash ${ }^{1}$, Jean-Michel Arbona ${ }^{2}$, Olivier Hyrien $^{3}$, Benjamin Audit ${ }^{4}$, Kathrin Marheineke*1 and Arach Goldar*1

\footnotetext{
${ }^{1}$ Institute of Integrative Biology of the Cell (I2BC), CNRS, CEA, University Paris Sud, 1, avenue de la Terrasse, 91190 Gif-sur-Yvette, France

${ }^{2}$ Univ. Lyon, ENS de Lyon, Univ. Claude Bernard Lyon 1, CNRS UMR5239, INSERM U1210,46 Allé d'Italie Site Jacques Monod, 69007 Lyon, France

${ }^{3}$ Institut de Biologie de l'Ecole normale supérieure (IBENS), Ecole normale supérieure, CNRS, INSERM, PSL Research University, 75005 Paris, France

${ }^{4}$ Univ. Lyon, ENS de Lyon, Univ. Claude Bernard Lyon 1, CNRS, Laboratoire de Physique, F-69342 Lyon, France

Email: Diletta Ciardo - ciardo@bio.ens.psl.eu; Olivier Haccard - olivier.haccard@i2bc.paris-saclay.fr; Hemalatha Narassimprakash hemalatha.narassimprakash@i2bc.paris-saclay.fr; Jean-Michel Arbona - jeanmichel.arbona@ens-lyon.fr; Olivier Hyrien -

hyrien@biologie.ens.fr; Benjamin Audit - benjamin.audit@ens-lyon.fr ; Kathrin Marheineke - kathrin.marheineke@i2bc.paris-saclay.fr; Arach Goldar - arach.goldar@cea.fr;

* Corresponding author
}

\section{Abstract}

Background: During cell division, the duplication of the genome starts at multiple positions called replication origins. Origin firing requires the interaction of rate-limiting factors with potential origins during the $\mathrm{S}$ (ynthesis)-phase of the cell cycle. Origins fire as synchronous clusters which is proposed to be regulated by the intra-S checkpoint.

Results: By modelling the unchallenged, the checkpoint-inhibited and the checkpoint protein Chk1 over-expressed replication pattern of single DNA molecules from Xenopus sperm chromatin replicated in egg extracts, we demonstrate that the quantitative modelling of data requires: 1) a segmentation of the genome into regions of low and high probability of origin firing; 2) that regions with high probability of origin firing escape intra-S checkpoint regulation and 3) the variability of the rate of DNA synthesis close to replication forks is a necessary ingredient that should be taken in to account in order to describe the dynamic of replication origin firing. 
Conclusions: This model implies that the observed origin clustering emerges from the apparent synchrony of origin firing in regions with high probability of origin firing and challenge the assumption that the intra-S checkpoint is the main regulator of origin clustering.

Availabily: The datasets supporting the conclusions of this article are from reference [1].

\section{Background}

Eukaryotic genomes are duplicated in a limited time during the $\mathrm{S}$ phase of each cell cycle. Replication starts at multiple origins that are activated (fired) at different times in $\mathrm{S}$ phase to establish two diverging replication forks that progress along and duplicate the DNA at fairly constant speed until they meet with converging forks originated from flanking origins [2,3]. The mechanisms that regulate the timing of origin firing remain largely unknown 4 [9].

The core motor component of the replicative helicase, the MCM2-7 complex, is loaded on chromatin from late mitosis until the end of G1 phase as an inactive head-to-head double hexamer (DH) to form a large excess of potential origins 10,11. During S phase, only a fraction of the MCM2-7 DHs are activated to form a pair of active Cdc45-MCM2-7-GINS (CMG) helicases and establish bidirectional replisomes 2 . MCM2-7 DHs that fail to fire are inactivated by forks emanating from neighboring fired origins [12. Origin firing requires S-phase cyclin-dependent kinase (CDK) and Dbf4-dependent kinase (DDK) activities as well as the CDK targets Sld2 and Sld3 and the replisome-maturation scaffolds Dpb11 and Sld7 in S. cerevisiae. The six initiation factors Sld2 (RecQ4 in Xenopus), Sld3 (Tresline in Xenopus), Dpb11 (TopBP1 in Xenopus), Dbf4 (Drf1 in Xenopus), Sld7 (MTBP in Xenopus) and Cdc45 are expressed at concentrations significantly lower than the MCM complex and core replisome components, suggesting that they may be rate-limiting for origin firing 13,14 . Among these six factors, Cdc45 is the only one to travel with the replication fork.

DNA replication initiates without sequence specificity in Xenopus eggs 15, 16, egg extracts 17,20 and early embryos 21,22] (for review see [23]). To understand how a lack of preferred sequences for replication initiation is compatible with a precise S-phase completion time, investigators have studied replication at the single DNA molecule level using the DNA combing technique 24 28] which contrast to population 
based approaches, that average replication characteristics. DNA combing technique reveals cell-to-cell differences in origin activation important for understanding how genomes are replicated during S-phase, these experiments did not detect a regular spacing of initiation events but revealed that the origin firing rate strongly increases from early to late replication intermediates, speeding up late replication stages 24 25. An observation that has been also confirmed in many other model organisms, including human cell lines [29].

A mathematical model based on the assumptions i) that the probability of firing of each replication origin can be replaced by the averaged probability of firing calculated over all degree of freedom of origin firing process (MCM2-7 DH density, genomic position, chromatin compaction, nucleosome density, etc named "mean-field hypothesis"), ii) that firing of origins are independent events and ii) that fork speed is constant was proposed [30]. This model allowed the extraction of a time-dependent rate of replication initiation, $I(t)$, from the measured eye lengths, gap lengths and eye-to-eye distances on combed DNA molecules (Fig 66a) 30]. The extracted $I(t)$ markedly increased during S phase. Simulations incorporating this extracted $I(t)$ reproduced the mean eye length, gap length and eye-to-eye distance, but the experimental eye-to-eye distance distribution appeared "peakier" than the simulated one 23. 31. Modulating origin firing propensity by the probability to form loops between forks and nearby potential origins resulted in a better fit to the data without affecting $I(t) 31$.

Importantly, experiments revealed that in Xenopus, like in other eukaryotes, replication eyes are not homogeneously distributed over the genome but tend to cluster 26 28]. First, a weak correlation between the sizes of neighbouring eyes was observed 26,28, 31, consistent with firing time correlations. Second, more molecules with no or multiple eyes than expected for spatially uniform initiations were observed in replicating DNA 28]. There are two potential, non-exclusive mechanisms for these spatiotemporal correlations. The first one, compatible with a mean-field hypothesis, is that activation of an origin stimulates nearby origins. The second one, no longer consistent with a mean-field hypothesis, is that the genome is segmented into multi-origin domains that replicate at different times in S phase. This second hypothesis has been explored numerically in human and has been shown to be compatible with the universal bell shaped $I(t)$ profile 32 .

Interestingly, experiments in Xenopus egg extracts revealed that intranuclear replication foci labelled early in one S phase colocalized with those labelled early in the next S phase, whereas the two labels did not coincide at the level of origins or origin clusters were examined [33]. Given the different characteristic sizes of timing domains (1-5 Mb) and origin clusters (50-100 kb) in the Xenopus system, it is possible that 
origin correlations reflect both a programmed replication timing of large domains and a more local origin cross-talk within domains.

It is now well accepted that the intra-S phase checkpoint regulates origin firing during both unperturbed and artificially perturbed S phase $[1,28,34,36$. DNA replication stress, through the activation of the S-phase checkpoint kinase Rad53, can inhibit origin firing by phosphorylating and inhibiting Sld3 and Dbf4 37. The metazoan functional analogue of Rad53 is Chk1. Experiments in human cells under low replication stress conditions showed that Chk1 inhibits the activation of new replication factories while allowing origin firing to continue within active factories [34]. Experiments using Xenopus egg extracts suggested that the checkpoint mainly adjusts the rate of DNA synthesis by staggering the firing time of origin clusters 28. Our first model for DNA replication in Xenopus egg extracts 38] (which combined time-dependent changes in the availability of a limiting replication factor, and a fork-density dependent affinity of this factor for potential origins) was used to model the regulation of DNA replication by the intra-S checkpoint [1]. We showed that even during an unperturbed S phase in Xenopus egg extracts, Chk1 inhibits origin firing away from but not near active forks [1. To account for the regulation of DNA replication by the intra-S checkpoint, we replaced the dependency of origin firing on fork density by a Chk1-dependent global inhibition of origin firing with local attenuation close to active forks as was proposed in other contexts 34,3941 . This model was able to simultaneously fit the $I(f)$ (the rate of origin firing expressed as a function of each molecule's replicated fraction $f$ ) of both a control and a UCN-01-inhibited Chk1 replication experiment 1]. However, in that work we did not push further the analysis to verify if our model was able to explain simultaneously $I(f)$ (temporal program) and the eye-to-eye distance distribution (spatial program).

In the present work, using numerical simulations, we quantitatively analysed both the temporal and spatial characteristics of genome replication as measured by DNA combing in the in vitro Xenopus system. Xenopus egg extracts have been successfully used since over three decades now to study DNA replication in metazoans 42 . Rooted on experimental data, we build a general and minimal model of DNA replication able to predict both the temporal and the spatial characteristics either during an unchallenged or a challenged S phase. We use the experimental data from [1] where the experimental mean chosen for activating or inhibiting (manipulating) the checkpoint was respectively to overexpress Chk1 protein or to inhibit its activity using the specific inhibitor UCN-01. By analysing the spatio-temporal pattern of DNA replication after inhibition or activation of intra-S checkpoint and comparing them to an unchallenged pattern we disentangle the complex role of the intra-S checkpoint for replication origin firing. 


\section{Results}

\section{Finding the best integrative model of unperturbed $S$ phase}

Our previous model [1] failed to simultaneously reproduce the eye-to-eye distance distribution and the $I(f)$ of the same control experiment (Fig 1). This discrepancy could be explained if initiation events have a strong tendency to cluster 26, 28]. Clustering produces an excess of small (intra-cluster) and large (inter-cluster) eye-to-eye distances compared to random initiations, but only the former could be detected on single DNA molecules due to their finite length [28. Chk1 action has been proposed to regulate origins clusters 34. However, Chk1 inhibition by UCN-01 did not result in the broader eye-to-eye distribution predicted by random origin firing ( Fig 1 c and d), suggesting that other mechanisms than intra-S checkpoint are involved in the origin clustering. We therefore explored the ability of several nested models

(a)

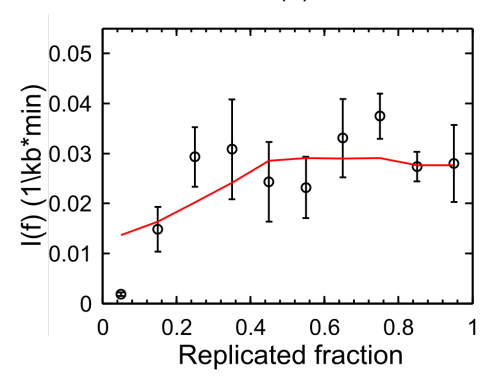

(c)

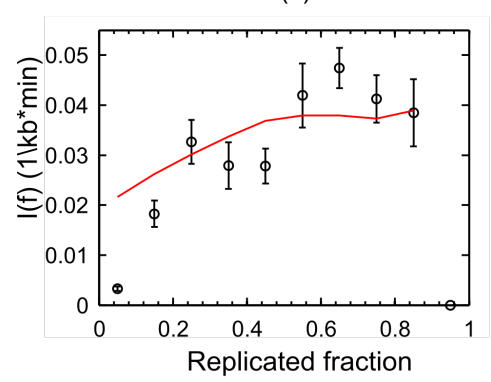

(b)

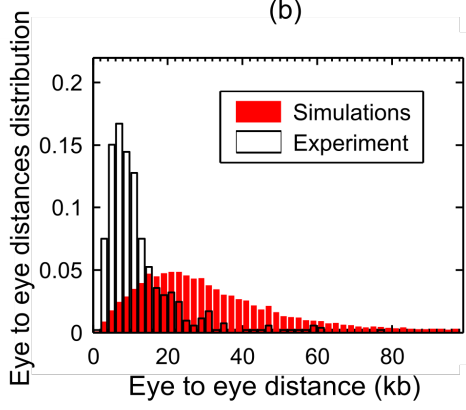

(d)

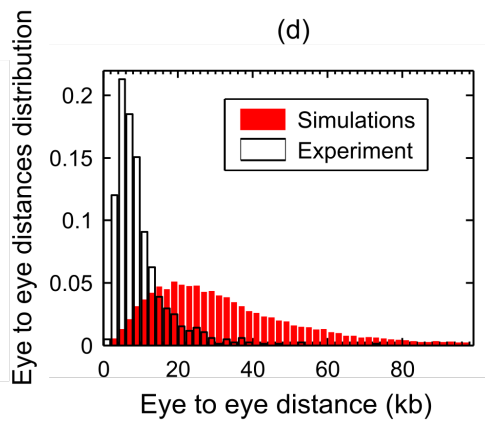

Figure 1: Chk1 does not control origin clustering. The black symbols are experimental data and the red curves are simulations. (a) and (c) Fitting of $I(f)$ data extracted from raw data published in [1] as described in material and methods for control and Chk1 inhibition experiments respectively. The discrepancy in values between the extracted data and those published in 11 are due to difference in thresholding and the lack of smoothing of the extracted data in this work. (b) and (d) Discrepancy between experimental and simulated distributions of eye-to-eye distances in control and Chk1 inhibition experiments, respectively.

with growing complexity (designated MM1 to MM5) Additional file 1 -Choosing the best model. . MM1 corresponds to a mean field hypothesis of origin firing : all potential origins have a constant firing probability $P_{\text {out }}$ 38, 43]. MM2 corresponds to MM1 but assuming that replication forks can have a variable speed 44, 45]. MM3 corresponds to MM1 with a local perturbation, whereby the proximity of forks 
facilitates origin firing [31, 46] over a distance $d$ downstream of an active fork where the probability of origin firing is $P_{\text {local }}$. In MM4 origin firing does not follow the mean field hypothesis but assumes that the genome can be segmented into regions of high and low probabilities of origin firing 32,46 as accepted for most eukaryotes $9,47,52$. In this scenario, the probability of origin firing of potential origins located within a fraction $\theta$ of the genome, $P_{i n}$, is assumed to be higher than the firing probability $P_{\text {out }}$ of potential origins in the complementary fraction $1-\theta$. Lastly, MM5 combines the specific features of MM3 and MM4 into a single model. Furthermore, to verify if the localized nature of potential origins 48,53 can influence the spatio-temporal program of origin firing, each considered scenario was simulated assuming either a continuous or a discrete distribution of potential origins except for MM2.

For each model, we coupled dynamic Monte Carlo numerical simulations to a genetic optimization algorithm to find the family of variables that maximized the similarity between the simulated and measured profiles of $I(f)$, replicated fraction of single molecules, global fork density, eye-to-eye distances, gap lengths and eye lengths. MM5 with localized potential origins (Fig 2 provided the best fit to the experimental data Additional file 1 - Choosing the best model. Figure 9). The increase in concordance between MM5 and the data occurs at the expense of increasing the number of parameters, which is justifiable on statistical grounds Additional file 1 - Choosing the best model.TABLE 2) and the predictive ability of MM5 is verified Additional file 2 - Testing the predictive capacity of MM5).

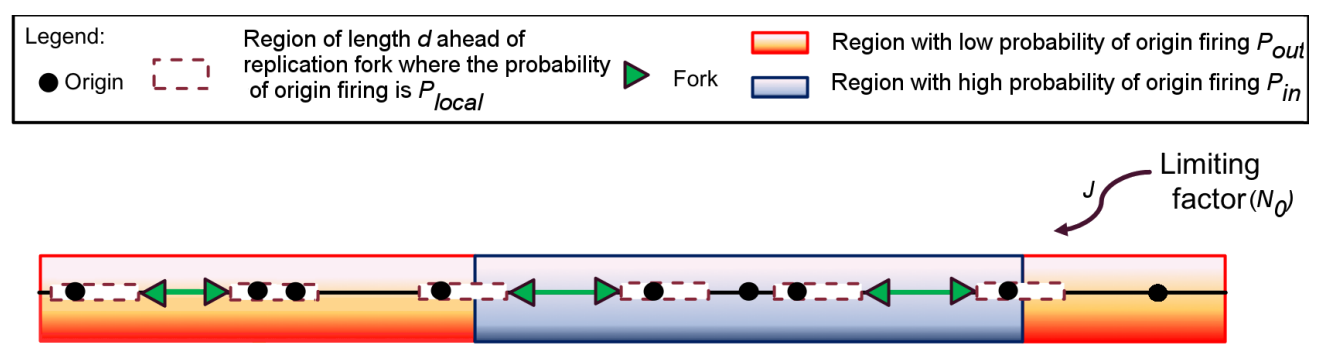

Figure 2: Schematic representation of MM5. Potential replication origins located in a fraction $\theta$ of the genome (not necessary contiguous) have a probability of firing $P_{\text {in }}$ higher than probability of firing $P_{\text {out }}$ of potential origins located in the complementary genome fraction $1-\theta$. The firing of a potential origins requires its encounter with limiting factors which number $N(t)=N_{0}+J t$ increases as $\mathrm{S}$ phase progresses. Potential origins fire with a probability $P_{\text {local }}$ over a distance $d$ ahead of a replication fork.

We used MM5 to analyse unchallenged, checkpoint inhibited and Chk1 over expressed S phase Additional file 3 -Fitting the experimental data with MM5). In all cases MM5 was able to model concomitantly $I(f)$ and eye-to-eye distance distribution (Fig 3). In conclusion, while MM5 does not include all the possible mechanisms involved in DNA replication process and its regulation, it can adequately predict the 
spatio-temporal dynamics of DNA replication and its regulation by checkpoint mechanisms using a limited number of processes.
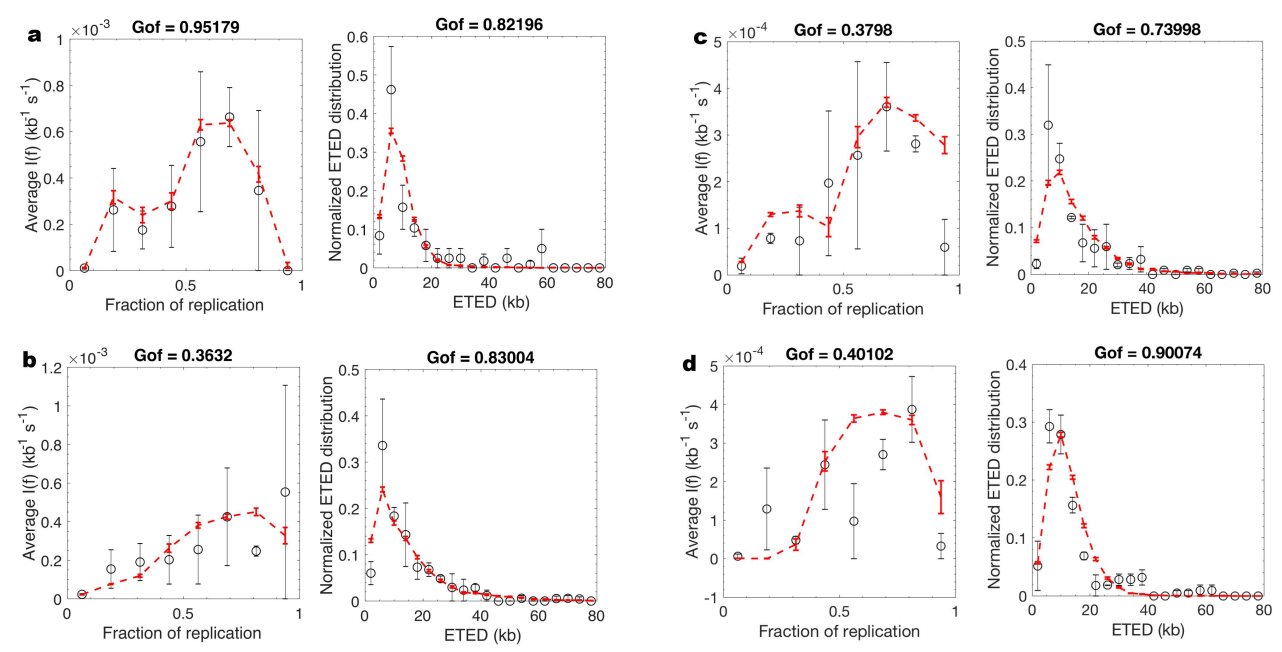

Figure 3: MM5 captures the essential processes necessary to model the regulation of DNA replication by Chk1. a and b. Unchallenged ( $8 \%$ global replication fraction) and Chk1 inhibited samples (22\% global replication fraction) corresponding to the same experiment and harvested at same time. $\mathbf{c}$ and d. Unchallenged ( $46 \%$ global replication fraction) and Chk1 over expressed samples ( $22 \%$ global replication fraction) corresponding to the same experiment and harvested at same time. The black open circles are experimental data and the dashed red lines are the fit obtained by MM5 model

\section{Retrieving the dynamics of an unchallenged S phase using the MM5 model}

MM5 faithfully reproduced the temporal and spatial program of DNA replication from unperturbed S phase samples with global replicated fractions of $8 \%, 19 \%$ and $53 \%$ Additional file 1 - Choosing the best model. Figure 9; Additional file 3-Fitting the experimental data with MM5, Figures 1 and 2). The fitted values of parameters changed as S phase progressed (Fig 4). However, only changes in $J, \theta, P_{\text {out }}$ and $d$ were statistically significant Additional file 3-Fitting the experimental data with MM5 Figure 3). In particular we found that $J$ increased from $8 \%$ to $19 \%$ replication and then dropped back at $53 \%$ replication. $\theta$ and $P_{\text {out }}$ increased only from $8 \%$ to $19 \%$ replication but not later, while $d$ stayed constant between $8 \%$ and $19 \%$ replication and decreased at $53 \%$ replication.

These observations suggest that during an unchallenged S phase both the fraction $(\theta)$ of the genome with high probability of origin firing and the background probability $\left(P_{\text {out }}\right)$ of origin firing outside that fraction increase as $\mathrm{S}$ phase progresses. Interestingly, $P_{\text {local }}$ is higher than $P_{i n}$ and $P_{\text {out }}$, suggesting that firing of an potential origin significantly favours the firing of nearby potential origins over a distance $d$, compatible 

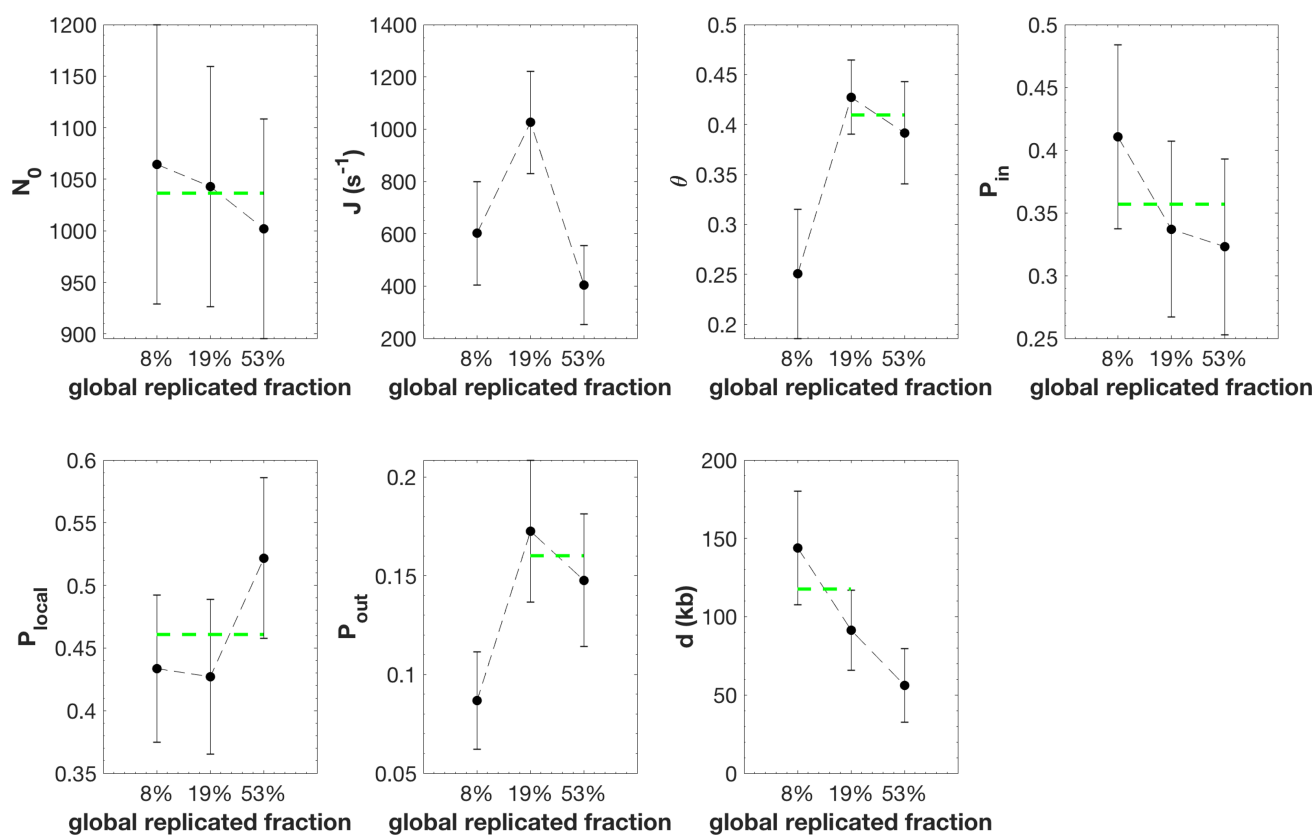

Figure 4: Inferred model parameters by fitting unchallenged $\mathbf{S}$ phase data as global replicated fraction increases. The black circles are the averaged value of the parameter over 100 independent fitting processes and the error bars are standard-deviations. The green dashed line is the mean value among consecutive parameters which differences are not statistically significant Additional file 3 -Fitting the experimental data with MM5 Figure 3).

with a chromatin looping process 46]. This fork-related firing process is consistent with the observation that nearby origins tend to fire at similar times, which has been proposed to result from a different regulation of nearby and distant origins by Chk1 [1,34.

\section{Modeling DNA replication under Chk1 inhibition and over expression}

To decipher the regulation of origin firing by Chk1, we examined if the MM5 model could also reproduce the replication program observed when the intra-S phase checkpoint was perturbed by the specific Chk1 inhibitor UCN-01 or by Chk1 over expression. We analyzed combed fibres from a replicated sample in the presence of UCN-01 (replicated fraction 22\%) and in Chk1 over expression condition (replicated fraction $22 \%$ ) that had spent the same interval of time in S phase as the control sample (global replicated fraction of $8 \%$ for UCN-01 and $46 \%$ in presence of Chk1 over expression). The MM5 model reproduced the experimental observations very well Additional file 3 -Fitting the experimental data with MM5, Figure 4 and Figure $7, G o F_{\text {global }}=0.85$ for $\mathrm{UCN}-01$ and $G o F_{\text {global }}=0.65$ for Chk1 over expression). The two parameters $J$ and $\theta$, were significantly higher in the UCN-01 treated sample than in the control samples 

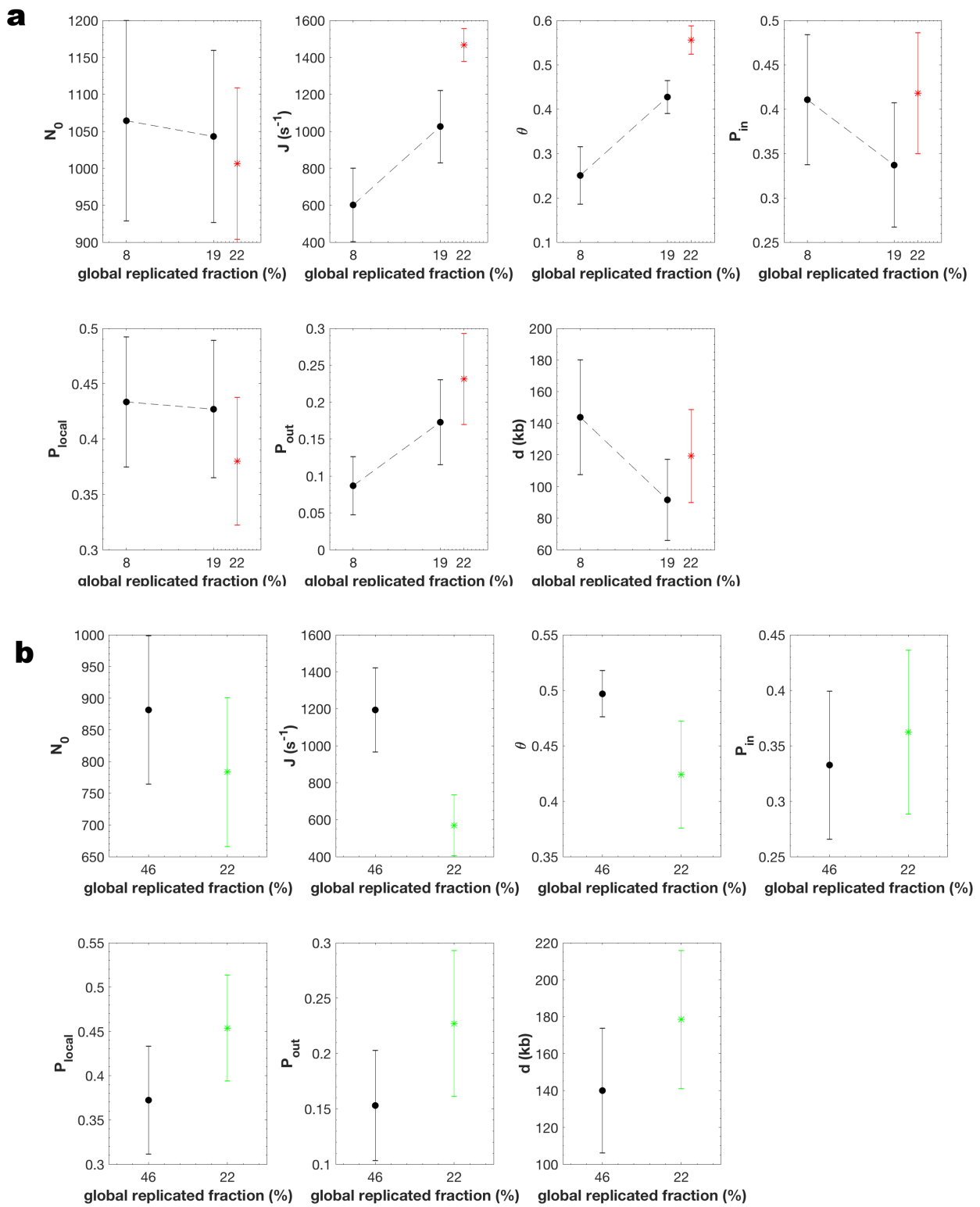

Figure 5: $J, \theta$, and the $P_{\text {out }}$ are the only parameters that change when comparing unchallenged, (a) Chk1 inhibited and (b)Chk1 over-expressed S phase The black circle is the averaged value of the parameter over 100 independent fitting processes of unchallenged S phase and the error bars are standarddeviations. The red star (a) is the averaged value of the parameter over 100 independent fitting processes of Chk1 inhibited sample and the error bars represent the standard-deviations. The green star (b) is the averaged value of the parameter over 100 independent fitting processes of Chk1 over-expressed sample and the error bars represent the standard-deviations.

with either the same harvesting time or a similar replicated fraction $(22 \%$ and $19 \%$, respectively) (Fig 5 and Additional file 3 -Fitting the experimental data with MM5 Figure 5). $P_{\text {out }}$ was higher in the UCN-01 
treated sample than in the control samples with the same harvesting time but unchanged once comparing similar replicated fraction. In the same manner, $J$, and $\theta$, were significantly lower in the Chk1 over expressed sample than in the control sample with the same incubation time (Fig 5 b and Additional file 3 -Fitting the experimental data with MM5 Figure 8). However, $P_{\text {out }}$ and the other parameters were unchanged compared to control samples.

MM5 belongs to the general family of KJMA models that probabilistically describes the state of a nucleating and growing system [54]. In this framework, probabilities describing the nucleation are analogous to the probabilities of origin firing [55] and their values only depend on the parameters that describe the state of the system that in our case only the global fraction of replicated DNA. Hence, It seems natural that for two samples with the same replication fraction the values of probabilities $P_{\text {out }}, P_{\text {in }}$ and $P_{\text {local }}$ remain unchanged.

These results suggest that upon Chk1 inhibition (i) a fraction $\theta$ of the genome, where initiation probability is high, increases during S phase; (ii) the probability of origin firing is insensitive to Chk1 within this fraction $\left(P_{\text {in }}\right.$ is unaltered) but is increased in the rest of the genome ( $P_{\text {out }}$ is increased) ; (iii) the import/activation rate of the limiting factor, $J$, is increased, while the starting number of factors, $N_{0}$, is unaffected. As was expected, MM5 detected that Chk1 inhibition by UCN-01 increased origin firing 1 [1, 35, 56 59. However, upon Chk1 over expression (i) the fraction $\theta$ of the genome decreases, (ii) $P_{\text {out }}$ is insensitive to Chk1 over expression and (ii) the import/activation rate of the limiting factor, $J$, is decreased, while the starting number of factors, $N_{0}$, is unaffected. As was expected, MM5 detected that Chk1 over expression reduced the number of fired origins [1].

In conclusion, the level of Chk1 appears to regulate the kinetics of S phase progression (i) by limiting the genome fraction that escapes its inhibitory action, (ii) by down regulating the probability of origin firing outside this fraction $[35,56,57,60]$ at the start of $\mathrm{S}$ phase, and (iii) by controlling the import/activation rate of limiting firing factors 35]. However, no significant differences in the strength of origin regulation by nearby forks $\left(P_{\text {local }}\right)$ was observed after Chk1 inhibition or over expression, suggesting that this local action is not mediated by Chk1 34 39].

\section{Discussion}

We explored several biologically plausible scenarios to understand the spatio-temporal organization of replication origin firing in Xenopus egg extracts. We used a quantitative approach to objectively discriminate which model best reproduced the genomic distributions of replication tracks as analyzed by 
DNA combing at different stages of S phase. We found that model MM5 with discrete potential origins best reproduced the experimental data with a minimal number of adjustable parameters. This model combines five assumptions [1, 30, 32, 38, $43,46,48,53,61,62$ : 1) origin firing is stochastic, 2) the availability of a rate-limiting firing factor captures the essential dynamics of the complex network of molecular interactions required for origin firing, 3) the speed of replication forks is constant 4) origins fire in a domino-like fashion in the proximity of active forks 46,$63 ; 5)$ the probability of origin firing is heterogeneous along the genome 32,48 .

We used MM5 to model DNA combing data from Xenopus egg extracts in presence or absence intra-S checkpoint inhibition and activation. In all conditions, this model was able to match the experimental data in a satisfactory manner. Furthermore, the inferred parameters values indicated that the global probability of origin firing and the rate of activation/import of the limiting firing factor $(J)$ were increased after Chk1 inhibition by UCN-01 $35,58,64$ and decreased after Chk1 over expression. Importantly, this model assumes a heterogeneous probability of origin firing and suggests that Chk1 exerts a global origin inhibitory action during unperturbed S phase [1] by following two possible mechanisms: (i) the first path corresponds to the regulation of the number of available replication limiting factors by Chk1 protein and (ii) the second path corresponds to the ability of Chk1 protein to reduce the capacity of potential origins to fire outside domains with high probability of origin firing. The strength of the second path decreases from the beginning of S phase to reach its minimal value after the first quarter of $\mathrm{S}$ phase. On the other hand, the constancy of the initial number of limiting factors $N_{0}$ in the presence or absence of UCN-01 or Chk1 over expression suggests that Chk1 does not actively control origins or the available number of replication limiting factors before $\mathrm{S}$ phase actually starts $36,65,66$. Interestingly, a better statistical match between the model and the data was obtained by assuming that the rate of DNA synthesis is variable downstream of replication forks. Indeed, the downstream of a replication forks the rate of DNA synthesis depends on the speed of replication fork and the frequency of firing of closeby potential replication origins 54. Our analysis suggests that this variability cannot be mapped to a model with variable fork speed, but it is compatible with an increased probability of origin firing in the neighbourhood of an active replication fork. These observations indicate that MM5 can deliver a reliable, minimally complex picture of origin firing regulation in Xenopus egg extracts. 


\section{The global inhibition of origin firing by Chk1}

We previously showed that Chk1 is active and limits the firing of some potential origins in an unperturbed S phase 11. Therefore, the earliest origins must be immune to Chk1 inhibition while later potential origins are strongly inhibited. The comparisons among the modelling of Chk1 inhibition, over expression and of unperturbed S phase data suggests that i) the probability of origin firing is reduced by active Chk1 in a fraction $1-\theta$ of the genome, ii) in this Chk1-sensitive fraction the probability of origin firing increases as $\mathrm{S}$ phase progresses and iii) the probability of origin firing is unaffected by Chk1 inhibition within the Chk1-immune, $\theta$ fraction of the genome. Therefore, this model supports the idea that at the start of $\mathrm{S}$ phase, some origins fire unimpeded by Chk1, whereas others remain silent. The latter only becomes progressively relieved from Chk1 inhibition as S phase progresses. Indeed, recent works in cultured mammalian cells 67, Drosophila [59 and Xenopus [68] showed that in unperturbed S phase the global origin firing inhibitory effect (by Chk1 and Rif1) is reduced as S phase progresses. Interestingly, a recent study in unperturbed yeast cells suggests that dNTPs are limiting at the entry into S phase, so that, similar to Xenopus [69], the firing of the earliest origins creates a replication stress that activates the Rad53 checkpoint which prevents further origin firing. Rad53 activation also stimulates dNTP synthesis, which in turn down regulates the checkpoint and allows later origin firing [36]. However, it remains uncertain if this feed-back loop does also exist in Xenopus egg extracts which contain an abundant pool of dNTPs. A key mechanism of our model is the enhancement of origin firing close to active forks. The necessity to introduce this mechanism supports the idea that the rate of DNA synthesis depends on the S-phase time and position of replication forks. During our modelling based on statistical ground we showed that the domino-like view of DNA replication progression 46,63 better describes the measured quantities from combed DNA molecules than the hypothesis of variable fork velocity 70 . It was previously shown in Xenopus egg extracts that the probability of origin firing could depend on the distance between left and right approaching forks 31. While this could in principle reflect an origin firing exclusion zone ahead of forks 24, 46, our model did not allow for a negative $P_{\text {local }}$ but the fact that the discrete distribution of potential replication origins better describes the experimental data than the continuous distribution confirm the necessity of the existence of origin firing exclusion zones between two converging replication forks. Other proposed mechanisms for origin clustering include the relief of Chk1 inhibition ahead of active forks by checkpoint recovery kinase polo like kinase 1 (Plk1) 1,39$]$. However, we find that the range, $d$, and the strength, $P_{\text {local }}$, of origin stimulation by nearby forks, were both insensitive to checkpoint inhibition or activation (Fig 5 a and b). Other potential mechanisms such as propagation of a supercoiling 
wave ahead of forks may better explain this insensitivity to Chk1 inhibition [71].

\section{Heterogeneous probability of origin firing}

In this model, the origin firing process in Xenopus egg extracts is not reliably described by a mean-field approximation. In other words, the probability of origin firing is heterogeneous along the genome. Based on this hypothesis, one important outcome of our study is that the genome can be segmented into domains where origin firing probability is either high and immune to Chk1 inhibition or low and subjected to a tight Chk1 control that attenuates as S phase progresses. This picture challenges the common view that the embryonic Xenopus in vitro system would lack the temporal regulation by the intra-S checkpoint at the level of large chromatin domains in contrast to findings in somatic vertebrate cells where Chk1 controls cluster or replication foci activation [60]. However, observations of replicating nuclei in Xenopus system have shown that early replication foci are conserved in successive replication cycles, supporting the heterogeneous domain hypothesis [33. Furthermore, we found that the fraction of the genome covered by these domains increases and that the inhibitory action of Chk1 decreases over time during an unperturbed S phase (Fig 4 and Fig 5), consistent with the idea that as S phase progresses more regions of the genome evade the checkpoint inhibition of origins. By comparing samples that have spent the same time interval in $\mathrm{S}$ phase or that have reached the same replicated fraction in the absence and presence of UCN-01 (Fig 5 a) or have spent the same time interval in S phase in a Chk1 over-expressed condition (Fig 5 b), we noticed that the probability of origin firing in the Chk1-immune domains $\left(P_{i n}\right)$ did not change upon Chk1 inhibition or over expression. This further suggests that these domains actually escape the regulation of origin firing by Chk1 that rules the rest of the genome. It is an interesting observation that in Chk1-immune regions where the probability of origin firing is high, the temporal difference between two firing events would be smaller than in other regions of the genome. This leads to an observed synchrony of origin firing and therefore to an effective observed clustering of replication eyes on a single DNA fibre.

\section{Conclusions}

All together the results of our modelling approach and the existing literature suggest that in the Xenopus system the position of early replicating, Chk1-immune domains is conserved in individual nucleus.

However, there is no experimental or numerical evidence that the positions of these domains are conserved in a population of nuclei. Assuming that the position of these domains changes randomly from one nucleus to another would result in a flat mean replication timing pattern and involves that each nucleus has its 
specific replication regulation process. While we cannot reject such a hypothesis objectively, the recent report of a structured replication timing program in zebrafish early embryos 52 encourages us also to assume the hypothesis that in Xenopus early embryos the position of early replication domains are conserved from one nucleus to another leading not to a flat but structured mean replication timing pattern similar to other eukaryotic systems $7,9,49]$.

\section{Methods}

\section{Monte Carlo simulation of DNA replication process.}

A dynamical Monte Carlo method was used to simulate the DNA replication process as detailed before [38]. We simulate the replicating genome as a one-dimensional lattice of $L=10^{6}$ blocks of value 1 for replicated and 0 for unreplicated state, respectively. To match the spatial resolution of DNA combing experiments each block represents $1 \mathrm{~kb}$. After one round of calculation an existing replication track grows in a symmetric manner by 2 blocks. Considering that the fork speed $v=0.5 \mathrm{~kb} \mathrm{~min}^{-1}$ is constant (except in MM2 where the value of $v\left(\mathrm{~kb}_{\mathrm{min}} \mathrm{m}^{-1}\right)$ for each active fork is randomly chosen at each round of calculus from $\{0,1,2,3\}\left(\mathrm{kb}_{\mathrm{min}} \mathrm{m}^{-1}\right)$ ), one round of calculation corresponds to 2 minutes. In the continuous case we assume that the potential replication origins are continuously distributed on the genome with an average density of one potential origin per $1 \mathrm{~kb}$ (1 block). As it is also considered that potential replication origins are discrete objects and as a consequence are distributed in a heterogeneous manner on the genome [48,53. we also simulate the case where the distribution of potential origins is discrete. In the discrete case we assume that potential origins are randomly distributed along the genome with an average density of one potential origin per $2.3 \mathrm{~kb} 72$. In both cases origins fire stochastically. Origin firing requires an encounter with a trans-acting factor which number $N(t)$ increases as S phase progresses with a rate $J$, $N(t)=N_{0}+J t$. If an encounter leads to an origin firing event, the trans-acting factor is sequestrated by replication forks and hence the number of available trans-acting factors is $N_{f}(t)=N(t)-N_{b}(t)$, where $N_{b}(t)$ is the number of bound factors. To ensure that origins do not re-fire during one cycle and are inactivated upon passive replication, only "0" blocks (not replicated) are able to fire. Before the beginning of replication process the one-dimensional lattice is randomly segmented into $\theta L$ blocks where the probability of origin firing is $P_{\text {in }}$ and $(1-\theta) L$ blocks where the probability of origin firing is $P_{\text {out }}$. After the start of replication process, at each round of calculus, each block is randomly assigned a value between 0 and 1. This value is compared to $P_{\text {in }}$ or $P_{\text {out }}$ (depending to which category the block belongs) to decide whether the block may fire. In total, $M$ " 0 " blocks $(M \leq L)$ with value strictly smaller than their 
reference probability may fire. If $M \leq N_{f}(t)$ all $M$ blocks may fire, otherwise $N_{f}(t)$ blocks may fire. Furthermore, in MM3 and MM5, we consider that the probability of origin firing $P_{\text {local }}$ may be increased downstream of a replication fork over a distance $d$. The trans-acting factors sequestered by forks are released and are made available for new initiation events when forks meet.

Measuring: the replicated fraction $f(t)$, the rate of origin firing $I(t)$, fork density $N_{f o r k}(t)$, eye-to-eye, eye and gap length distributions.

The genome is represented as a one-dimensional lattice of $10^{6}$ elements $x_{i} \in\{0,1\}$. At each round of calculation the replicated fraction is calculated as $f(t)=\langle x\rangle_{i}$ corresponding to the average value of $x_{i}$ over the genome.

The rate of origin firing per length of unreplicated genome per time unit $(3 \mathrm{~min})$ is calculated at each

round of calculation, by counting the number of newly created "1" blocks, $N_{1}$ and $I(t)=\frac{N_{1}}{(1-f(t)) L \Delta t}$ where $\Delta t=3 \mathrm{~min}$ and $L=10^{6}$. The density of replication forks is calculated at each round of calculation by counting the number of "01" tracks, $N_{\text {left }}$, and "10" tracks, $N_{\text {right }}$ and $N_{\text {forks }}(t)=\frac{N_{\text {right }}+N_{\text {left }}}{L}$. The distributions of eye-to-eye distances, eye lengths and unreplicated gap sizes are then computed from the distribution of "0" and "1" tracks after reshaping the data (see below).

\section{Comparing experimental and numerical data.}

The simulation results were compared to the DNA combing data from Platel et al. 11. The fluorescence intensities for total DNA and replicated tracks of each fiber were measured and binarized on a Matlab ${ }^{\circledR}$ platform by using a thresholding algorithm. The threshold value was chosen to minimize the difference between the replicated fraction measured by $\alpha^{32} \mathrm{P}-\mathrm{dATP}$ incorporation and by DNA combing. Replicated tracks larger than $1 \mathrm{~kb}$ were scored as eyes. Gaps were considered significant if $>1 \mathrm{~kb}$, otherwise the two adjacent eyes were merged. The eyes whose lengths span from 1 to $3 \mathrm{~kb}$ were considered as new origin firing events. The time interval in which these new detectable events can occur was calculated as $\Delta t=3 \mathrm{~min}$ assuming a constant replication fork velocity of $v \approx 0.5 \mathrm{~kb} \cdot \mathrm{min}^{-1}$. This data reshaping protocol was also applied to simulated DNA molecules, in order to match the spatial and temporal resolutions between the experimental and simulated data. The global replicated fraction of each sample was computed as the sum of all eye lengths divided by the sum of all molecule lengths. To minimize finite molecule length effects in comparisons between data and simulations, the experimental molecule length distribution was normalised and considered as probability density of molecule length in the sample and 
used to weight the random shredding of the simulated genome at each time (Fig 6 b). The global replication fraction of simulated cut molecules was calculated. Only molecules from the simulation time that had the same global replication fraction as the experimental sample were further considered.

$$
\text { a }
$$

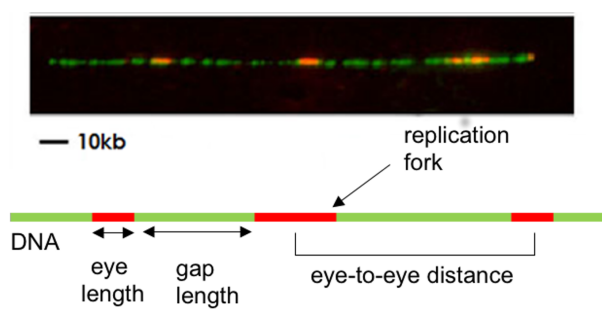

Figure 6: Characteristics of combed DNA molecules. a. Example of combed DNA molecule. The top panel is a fluorescence microscopy of a representative, stretched DNA fiber (green) containing replication eyes (red). The bottom panel is a schematic illustration of measured parameters in replication studies using DNA combing. b. Molecular length distribution (global replicated fraction of 8\%) of combed DNA fibre. The black open circles are the experimentally measured and the red curve is the simulated cut molecular length distributions, respectively.

Molecules were sorted by replicated fraction $f(t)$. The rate of origin firing and fork density were calculated for each molecule as a function of $f(t)\left(I(f)\right.$ and $N_{\text {fork }}(f)$, respectively) for both simulated and experimental data. The experimental $I(f), N_{\text {fork }}(f)$, eye-to-eye distances, eye and gap length distributions were computed as the averaged value of three independent experiments.

\section{Modeling experimental data: parameters optimization.}

To estimate the parameters of the model, we fitted the six experimental observables $\left(I(f), N_{\text {fork }}(f)\right.$, replicated fibre, eye-to-eye distances, eye and gap length distribution) using a genetic optimization algorithm $\left(\right.$ Matlab $\left.{ }^{\circledR}\right)$. The fitness function was defined as the sum of the square of the differences between experimental and simulated data curves divided by the squared mean of the experimental data curve. The genetic optimization algorithm was set over three subpopulations of 20 individuals with a migration fraction of 0.1 and a migration interval of 5 steps. Each individual defined a set of variables for the 
simulation and the variables were chosen within the range reported in Table 1 for the model that best fit the data. At each generation, 3 elite children were selected for the next generation. The rest of the population corresponds to a mixture between $60 \%$ of children obtained after a scattered crossover between two individuals selected by roulette wheel selection and $40 \%$ of children obtained by uniform mutation with a probability of 0.2 , leading to a variability of $8 \%$. The genetic algorithm was stopped after 50 generations corresponding to the convergence of the optimization method. As the size of variable space is unknown, we considered a large domain of validity for the variables. This has as an effect to reduce the probability that the optimization process reaches a unique global minimum. For this reason we repeat the genetic optimization method 100 times independently over each data set and consider for each optimization round only the best elite individual.

Table 1: Lower and upper bounds of adjustable variables.

\begin{tabular}{lccl}
\hline Variable & Lower bound & Upper bound & Significance \\
\hline$N_{0}$ & 1 & 2000 & Initial number of limiting-factor \\
$J\left(s^{-1}\right)$ & 0 & 4000 & Rate at which the number of limiting-factor increases \\
$P_{\text {out }}$ & 0 & 1 & Probability of origin firing in the $1-\theta$ fraction \\
$P_{\text {in }}$ & 0 & 1 & Probability of origin firing in the $\theta$ fraction \\
$P_{\text {local }}$ & 0 & 1 & Probability of origin firing ahead of an active replication fork over a distance $d$ \\
$\theta$ & 0 & 1 & Fraction of genome where the probability of origin firing is $P_{i n}$ \\
$d(k b)$ & 0 & 1000 & Distance over which a fork acts on the probability of origin firing \\
\hline
\end{tabular}

\section{Authors contributions}

D.C., O.H., H.N, K.M and A.G. conceived of the presented idea. D.C and A.G developed the numerical simulations and performed the computations. J-M.A., O.H. and B.A. verified the methods. K.M. and A.G. supervised the findings of this work. All authors discussed the results and contributed to the final manuscript.

\section{Competing interests}

The authors declare that they have no competing interests.

\section{Acknowledgements}

This article is dedicated to the memory of our colleague and friend Alain Arneodo, who passed away during its elaboration and writing. The authors acknowledge Alain's enthusiasm and constant support. This work was supported by the Fondation pour la Recherche Médicale [FRM DEI201512344404], the 
Centre National de Recherche Scientifique (CNRS), the department of genome biology of I2BC, by a PhD fellowship of IdEX Paris-Saclay university, the Commissariat à l'énergie atomique (CEA) and the Cancéropole Ile-de-France [PLBIO16-302].

\section{References}

1. Platel M, Goldar A, Wiggins JM, Barbosa P, Libeau P, Priam P, Narassimprakash H, Grodzenski X, Marheineke K: Tight Chk1 Levels Control Replication Cluster Activation in Xenopus. PLoS One $2015, \mathbf{1 0}(6)$.

2. DePamphilis M, Bell SD: Genome Duplication. London ; New York: Garland Science 2010.

3. Machida YJ, Hamlin JL, Dutta A: Right Place, Right Time, and Only Once: Replication Initiation in Metazoans. Cell 2005, 123:13-24.

4. Raghuraman MK: Replication Dynamics of the Yeast Genome. Science 2001, 294(5540):115-121.

5. Heichinger C, Penkett CJ, Bähler J, Nurse P: Genome-wide Characterization of Fission Yeast DNA Replication Origins. EMBO J 2006, 25(21):5171-5179.

6. Eshaghi M, Karuturi RKM, Li J, Chu Z, Liu ET, Liu J: Global Profiling of DNA Replication Timing and Efficiency Reveals That Efficient Replication/Firing Occurs Late during S-Phase in S. Pombe. PLoS One 2007, 2(8).

7. Baker A, Audit B, Chen CL, Moindrot B, Leleu A, Guilbaud G, Rappailles A, Vaillant C, Goldar A, Mongelard F, d'Aubenton-Carafa Y, Hyrien O, Thermes C, Arneodo A: Replication Fork Polarity Gradients Revealed by Megabase-Sized U-Shaped Replication Timing Domains in Human Cell Lines. PLoS Comput Biol 2012, 8(4).

8. Audit B, Baker A, Chen CL, Rappailles A, Guilbaud G, Julienne H, Goldar A, d'Aubenton-Carafa Y, Hyrien O, Thermes C, Arneodo A: Multiscale Analysis of Genome-Wide Replication Timing Profiles Using a Wavelet-Based Signal-Processing Algorithm. Nat Protoc 2013, 8:98-110.

9. Rhind N, Gilbert DM: DNA Replication Timing. Cold Spring Harb Perspect Biol 2013, 5(8):a010132.

10. DePamphilis ML, Blow JJ, Ghosh S, Saha T, Noguchi K, Vassilev A: Regulating the Licensing of DNA Replication Origins in Metazoa. Current Opinion in Cell Biology 2006, 18(3):231-239.

11. Ticau S, Friedman LJ, Ivica NA, Gelles J, Bell SP: Single-Molecule Studies of Origin Licensing Reveal Mechanisms Ensuring Bidirectional Helicase Loading. Cell 2015, 161(3):513-525.

12. Blow JJ, Ge XQ, Jackson DA: How Dormant Origins Promote Complete Genome Replication. Trends Biochem Sci 2011, 36(8):405-414.

13. Mantiero D, Mackenzie A, Donaldson A, Zegerman P: Limiting Replication Initiation Factors Execute the Temporal Programme of Origin Firing in Budding Yeast. EMBO J 2011, 30(23):4805-4814.

14. Tanaka S, Nakato R, Katou Y, Shirahige K, Araki H: Origin Association of Sld3, Sld7, and Cdc45 Proteins Is a Key Step for Determination of Origin-Firing Timing. Current Biology 2011, 21(24):2055-2063.

15. Harland RM, Laskey RA: Regulated Replication of DNA Microinjected into Eggs of Xenopus Laevis. Cell 1980, 21(3):761-771.

16. Méchali M, Kearsey S: Lack of Specific Sequence Requirement for DNA Replication in Xenopus Eggs Compared with High Sequence Specificity in Yeast. Cell 1984, 38:55-64.

17. Mahbubani HM, Paull T, Elder JK, Blow JJ: DNA Replication Initiates at Multiple Sites on Plasmid DNA in Xenopus Egg Extracts. Nucleic Acids Res 1992, 20(7):1457-1462.

18. Hyrien O, Méchali M: Plasmid Replication in Xenopus Eggs and Egg Extracts: A 2D Gel Electrophoretic Analysis. Nucleic Acids Res 1992, 20(7):1463-1469.

19. Carli FD, Gaggioli V, Millot GA, Hyrien O: Single-Molecule, Antibody-Free Fluorescent Visualisation of Replication Tracts along Barcoded DNA Molecules. Int. J. Dev. Biol. 2016, 60(7-8-9):297-304. 
20. Carli FD, Menezes N, Berrabah W, Barbe V, Genovesio A, Hyrien O: High-Throughput Optical Mapping of Replicating DNA. Small Methods 2018, 2(9):1800146.

21. Hyrien O, Méchali M: Chromosomal Replication Initiates and Terminates at Random Sequences but at Regular Intervals in the Ribosomal DNA of Xenopus Early Embryos. The EMBO Journal 1993, 12(12):4511-4520.

22. Hyrien O, Maric C, Méchali M: Transition in Specification of Embryonic Metazoan DNA Replication Origins. Science 1995, 270(5238):994-997.

23. Hyrien O, Marheineke K, Goldar A: Paradoxes of Eukaryotic DNA Replication: MCM Proteins and the Random Completion Problem. Bioessays 2003, 25(2):116-125.

24. Lucas I, Chevrier-Miller M, Sogo JM, Hyrien O: Mechanisms Ensuring Rapid and Complete DNA Replication despite Random Initiation in Xenopus Early embryos11Edited by M. Yaniv. Journal of Molecular Biology 2000, 296(3):769-786.

25. Herrick J, Stanislawski P, Hyrien O, Bensimon A: Replication Fork Density Increases during DNA Synthesis in X. Laevis Egg extracts11Edited by M. Yaniv. Journal of Molecular Biology 2000, 300(5):1133-1142.

26. Blow JJ, Gillespie PJ, Francis D, Jackson DA: Replication Origins in XenopusEgg Extract Are 5-15 Kilobases Apart and Are Activated in Clusters That Fire at Different Times. J Cell Biol 2001, 152:15-26.

27. Marheineke K, Hyrien O: Aphidicolin Triggers a Block to Replication Origin Firing inXenopus Egg Extracts. J. Biol. Chem. 2001, 276(20):17092-17100.

28. Marheineke K, Hyrien O: Control of Replication Origin Density and Firing Time in Xenopus Egg Extracts ROLE OF A CAFFEINE-SENSITIVE, ATR-DEPENDENT CHECKPOINT. J. Biol. Chem. 2004, 279(27):28071-28081.

29. Goldar A, Marsolier-Kergoat MC, Hyrien O: Universal Temporal Profile of Replication Origin Activation in Eukaryotes. PLOS ONE 2009, 4(6):e5899.

30. Herrick J, Jun S, Bechhoefer J, Bensimon A: Kinetic Model of DNA Replication in Eukaryotic Organisms. Journal of Molecular Biology 2002, 320(4):741-750.

31. Jun S, Herrick J, Bensimon A, Bechhoefer J: Persistence Length of Chromatin Determines Origin Spacing in Xenopus Early-Embryo DNA Replication: Quantitative Comparisons between Theory and Experiment. Cell Cycle 2004, 3(2):223-229.

32. Gindin Y, Valenzuela MS, Aladjem MI, Meltzer PS, Bilke S: A Chromatin Structure-Based Model Accurately Predicts DNA Replication Timing in Human Cells. Mol Syst Biol 2014, 10(3):722.

33. Labit H, Perewoska I, Germe T, Hyrien O, Marheineke K: DNA Replication Timing Is Deterministic at the Level of Chromosomal Domains but Stochastic at the Level of Replicons in Xenopus Egg Extracts. Nucleic Acids Research 2008, 36(17):5623-5634.

34. Ge XQ, Blow JJ: Chk1 Inhibits Replication Factory Activation but Allows Dormant Origin Firing in Existing Factories. The Journal of Cell Biology 2010, 191(7):1285-1297.

35. Guo C, Kumagai A, Schlacher K, Shevchenko A, Shevchenko A, Dunphy WG: Interaction of Chk1 with Treslin Negatively Regulates the Initiation of Chromosomal DNA Replication. Molecular Cell 2015, 57(3):492-505.

36. Forey R, Poveda A, Sharma S, Barthe A, Padioleau I, Renard C, Lambert R, Skrzypczak M, Ginalski K, Lengronne A, Chabes A, Pardo B, Pasero P: Mec1 Is Activated at the Onset of Normal S Phase by Low-dNTP Pools Impeding DNA Replication. Molecular Cell 2020.

37. Zegerman P, Diffley JFX: Checkpoint Dependent Inhibition of DNA Replication Initiation by Sld3 and Dbf4 Phosphorylation. Nature 2010, 467(7314):474-478.

38. Goldar A, Labit H, Marheineke K, Hyrien O: A Dynamic Stochastic Model for DNA Replication Initiation in Early Embryos. PLoS ONE 2008, 3(8):e2919.

39. Trenz K, Errico A, Costanzo V: Plx1 Is Required for Chromosomal DNA Replication under Stressful Conditions. The EMBO Journal 2008, 27(6):876-885. 
40. Dimitrova DS, Gilbert DM: Temporally Coordinated Assembly and Disassembly of Replication Factories in the Absence of DNA Synthesis. Nat Cell Biol 2000, 2(10):686-694.

41. Thomson AM, Gillespie PJ, Blow JJ: Replication Factory Activation Can Be Decoupled from the Replication Timing Program by Modulating Cdk Levels. The Journal of Cell Biology 2010, 188(2):209-221.

42. Hoogenboom WS, Klein Douwel D, Knipscheer P: Xenopus Egg Extract: A Powerful Tool to Study Genome Maintenance Mechanisms. Developmental Biology 2017, 428(2):300-309.

43. Gauthier MG, Bechhoefer J: Control of DNA Replication by Anomalous Reaction-Diffusion Kinetics. Phys. Rev. Lett. 2009, 102(15):158104.

44. Conti C, Saccà B, Herrick J, Lalou C, Pommier Y, Bensimon A: Replication Fork Velocities at Adjacent Replication Origins Are Coordinately Modified during DNA Replication in Human Cells. Mol Biol Cell 2007, 18(8):3059-3067.

45. Yousefi R, Rowicka M: Stochasticity of Replication Forks' Speeds Plays a Key Role in the Dynamics of DNA Replication. PLOS Computational Biology 2019, 15(12):e1007519.

46. Löb D, Lengert N, Chagin VO, Reinhart M, Casas-Delucchi CS, Cardoso MC, Drossel B: 3D Replicon Distributions Arise from Stochastic Initiation and Domino-like DNA Replication Progression. Nature Communications 2016, 7:11207.

47. McCune HJ, Danielson LS, Alvino GM, Collingwood D, Delrow JJ, Fangman WL, Brewer BJ, Raghuraman MK: The Temporal Program of Chromosome Replication: Genomewide Replication in clb5 $\Delta$ Saccharomyces Cerevisiae. Genetics 2008, 180(4):1833-1847.

48. Yang SCH, Rhind N, Bechhoefer J: Modeling Genome-Wide Replication Kinetics Reveals a Mechanism for Regulation of Replication Timing. Molecular Systems Biology 2010, 6:404.

49. Boulos RE, Drillon G, Argoul F, Arneodo A, Audit B: Structural Organization of Human Replication Timing Domains. FEBS Letters 2015, 589(20, Part A):2944-2957.

50. Das SP, Borrman T, Liu VW, Yang SCH, Bechhoefer J, Rhind N: Replication Timing Is Regulated by the Number of MCMs Loaded at Origins. Genome Res 2015, 25(12):1886-1892.

51. Petryk N, Kahli M, d'Aubenton-Carafa Y, Jaszczyszyn Y, Shen Y, Silvain M, Thermes C, Chen CL, Hyrien O: Replication Landscape of the Human Genome. Nat Commun 2016, 7:10208.

52. Siefert JC, Georgescu C, Wren JD, Koren A, Sansam CL: DNA Replication Timing during Development Anticipates Transcriptional Programs and Parallels Enhancer Activation. Genome Res. 2017, 27(8):1406-1416.

53. Arbona JM, Goldar A, Hyrien O, Arneodo A, Audit B: The Eukaryotic Bell-Shaped Temporal Rate of DNA Replication Origin Firing Emanates from a Balance between Origin Activation and Passivation. eLife 2018, 7:e35192.

54. Jun S, Bechhoefer J: Nucleation and Growth in One Dimension. II. Application to DNA Replication Kinetics. Phys. Rev. E 2005, 71:011909.

55. Jun S, Zhang H, Bechhoefer J: Nucleation and Growth in One Dimension. I. The Generalized Kolmogorov-Johnson-Mehl-Avrami Model. Phys. Rev. E 2005, 71:011908.

56. Syljuasen RG, Sorensen CS, Hansen LT, Fugger K, Lundin C, Johansson F, Helleday T, Sehested M, Lukas J, Bartek J: Inhibition of Human Chk1 Causes Increased Initiation of DNA Replication, Phosphorylation of ATR Targets, and DNA Breakage. MCB 2005, 25(9):3553-3562.

57. Michelena J, Gatti M, Teloni F, Imhof R, Altmeyer M: Basal CHK1 Activity Safeguards Its Stability to Maintain Intrinsic S-Phase Checkpoint Functions. J. Cell Biol. 2019, 218(9):2865-2875.

58. Pommier Y, Kohn KW: Cycle cellulaire et points de contrôle en oncologie : nouvelles cibles thérapeutiques. Med Sci (Paris) 2003, 19(2):173-186.

59. Deneke VE, Melbinger A, Vergassola M, Di Talia S: Waves of Cdk1 Activity in S Phase Synchronize the Cell Cycle in Drosophila Embryos. Developmental Cell 2016, 38(4):399-412.

60. Maya-Mendoza A, Petermann E, Gillespie DA, Caldecott KW, Jackson DA: Chk1 Regulates the Density of Active Replication Origins during the Vertebrate S Phase. EMBO J 2007, 26(11):2719-2731. 
61. Blow JJ, Ge XQ: A Model for DNA Replication Showing How Dormant Origins Safeguard against Replication Fork Failure. EMBO Rep 2009, 10(4):406-412.

62. Sekedat MD, Fenyö D, Rogers RS, Tackett AJ, Aitchison JD, Chait BT: GINS Motion Reveals Replication Fork Progression Is Remarkably Uniform throughout the Yeast Genome. Molecular Systems Biology 2010, 6.

63. Guilbaud G, Rappailles A, Baker A, Chen CL, Arneodo A, Goldar A, d'Aubenton-Carafa Y, Thermes C, Audit B, Hyrien O: Evidence for Sequential and Increasing Activation of Replication Origins along Replication Timing Gradients in the Human Genome. PLOS Computational Biology 2011, 7(12):e1002322.

64. Seiler JA, Conti C, Syed A, Aladjem MI, Pommier Y: The Intra-S-Phase Checkpoint Affects Both DNA Replication Initiation and Elongation: Single-Cell and -DNA Fiber Analyses. MCB 2007, 27(16):5806-5818.

65. Lupardus PJ, Byun T, Yee Mc, Hekmat-Nejad M, Cimprich KA: A Requirement for Replication in Activation of the ATR-Dependent DNA Damage Checkpoint. Genes Dev. 2002, 16(18):2327-2332.

66. Stokes MP, Van Hatten R, Lindsay HD, Michael WM: DNA Replication Is Required for the Checkpoint Response to Damaged DNA in Xenopus Egg Extracts. J Cell Biol 2002, 158(5):863-872.

67. Moiseeva TN, Yin Y, Calderon MJ, Qian C, Schamus-Haynes S, Sugitani N, Osmanbeyoglu HU, Rothenberg E, Watkins SC, Bakkenist CJ: An ATR and CHK1 Kinase Signaling Mechanism That Limits Origin Firing during Unperturbed DNA Replication. PNAS 2019, 116(27):13374-13383.

68. Krasinska L, Besnard E, Cot E, Dohet C, Méchali M, Lemaitre JM, Fisher D: Cdk1 and Cdk2 Activity Levels Determine the Efficiency of Replication Origin Firing in Xenopus. The EMBO Journal 2008, 27(5):758-769.

69. Zou L: Single- and Double-Stranded DNA: Building a Trigger of ATR-Mediated DNA Damage Response. Genes Dev. 2007, 21(8):879-885.

70. Ciardo D, Goldar A, Marheineke K: On the Interplay of the DNA Replication Program and the Intra-S Phase Checkpoint Pathway. Genes 2019, 10(2):94.

71. Achar YJ, Adhil M, Choudhary R, Gilbert N, Foiani M: Negative Supercoil at Gene Boundaries Modulates Gene Topology. Nature 2020, 577(7792):701-705.

72. Edwards MC, Tutter AV, Cvetic C, Gilbert CH, Prokhorova TA, Walter JC: MCM2-7 Complexes Bind Chromatin in a Distributed Pattern Surrounding the Origin Recognition Complex in Xenopus Egg Extracts. J. Biol. Chem. 2002, 277(36):33049-33057.

\section{Additional Files}

Additional file 1 -Choosing the best model..

In this appendix we describe in detail why MM5 is statistically the best model to describe the data.

Additional file 2 - Testing the predictive capacity of MM5.

In this appendix we test the predictive capacity of the fitting procedure coupled to MM5.

\section{Additional file 3 -Fitting the experimental data with MM5.}

In this appendix we extract the values of free parameters from experimental data for unchalanged, Chk1 inhibited and Chk1 over-expressed S phase and discuss their variations. 

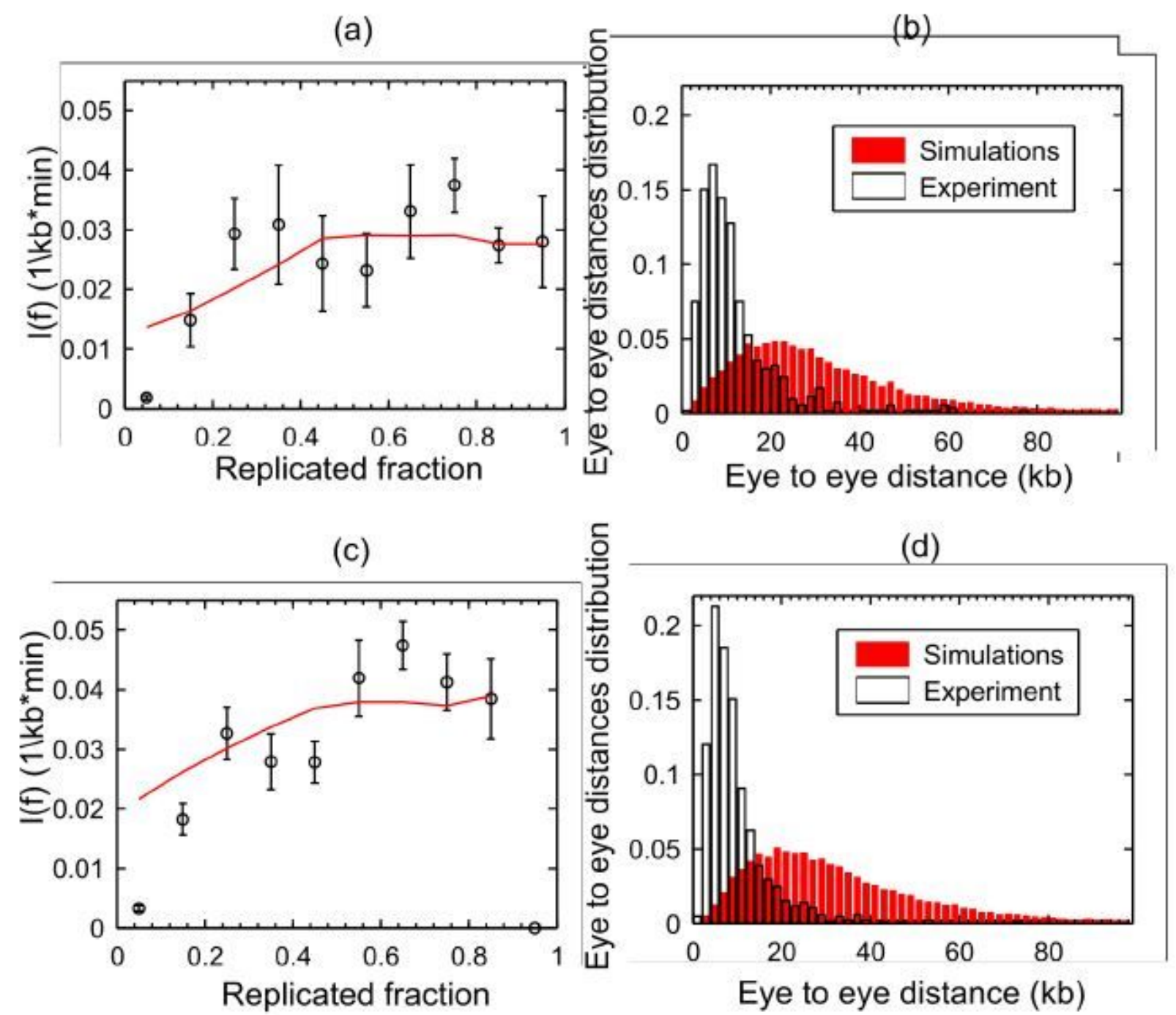

Figure 1

"Please see the Manuscript PDF file for the complete figure caption".

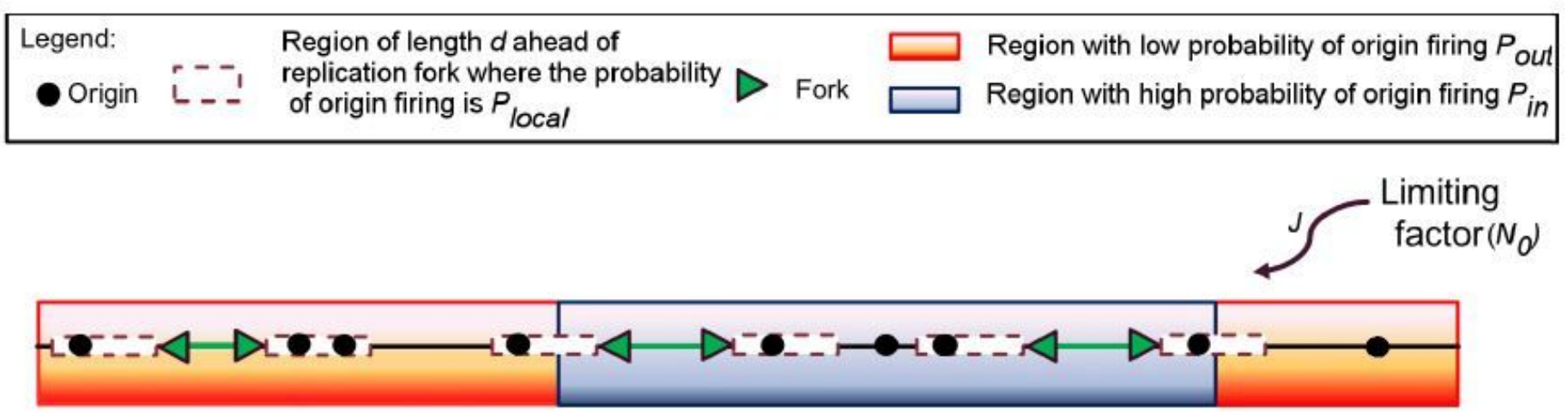

Figure 2 
"Please see the Manuscript PDF file for the complete figure caption".
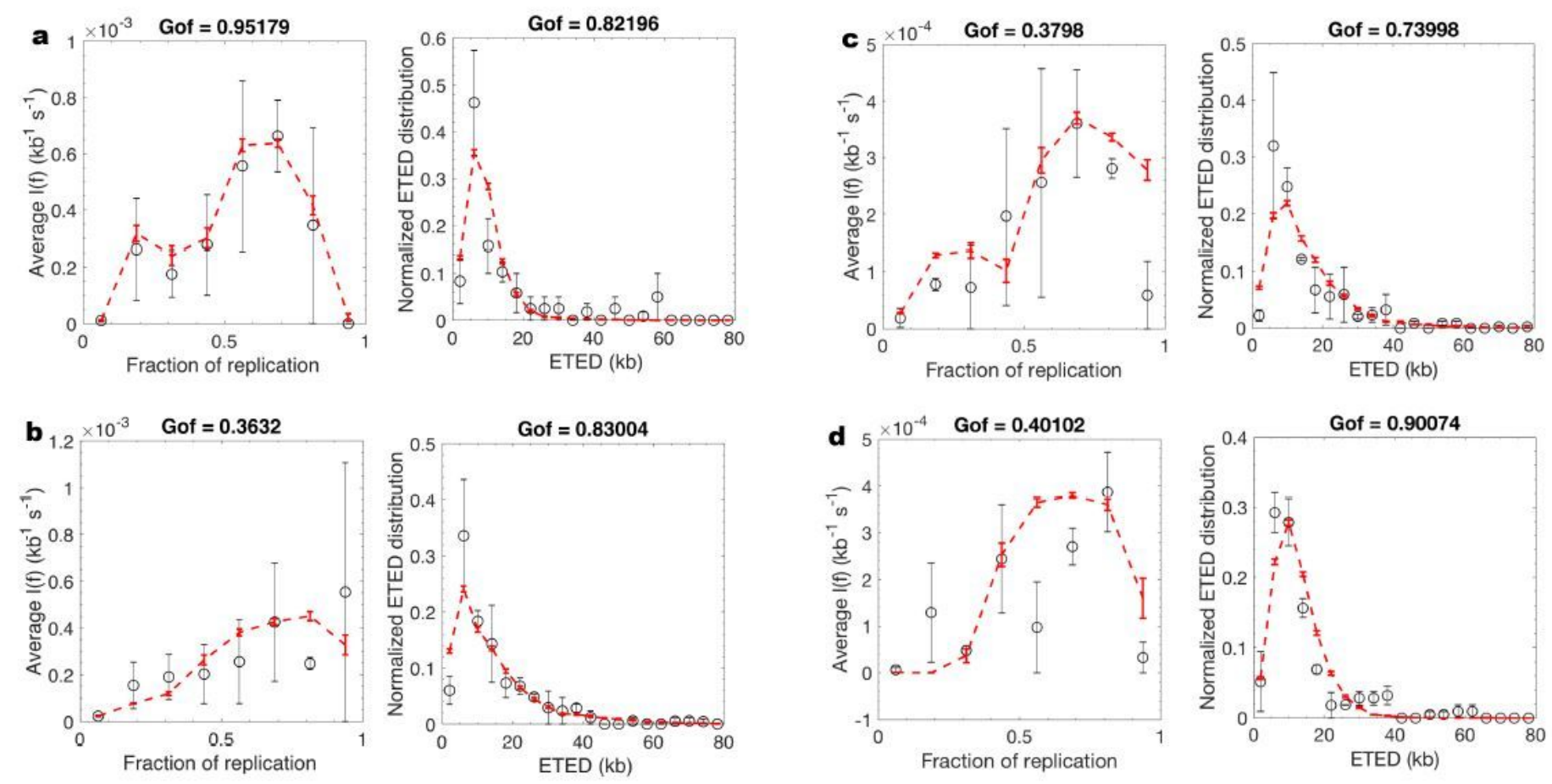

\section{Figure 3}

"Please see the Manuscript PDF file for the complete figure caption". 

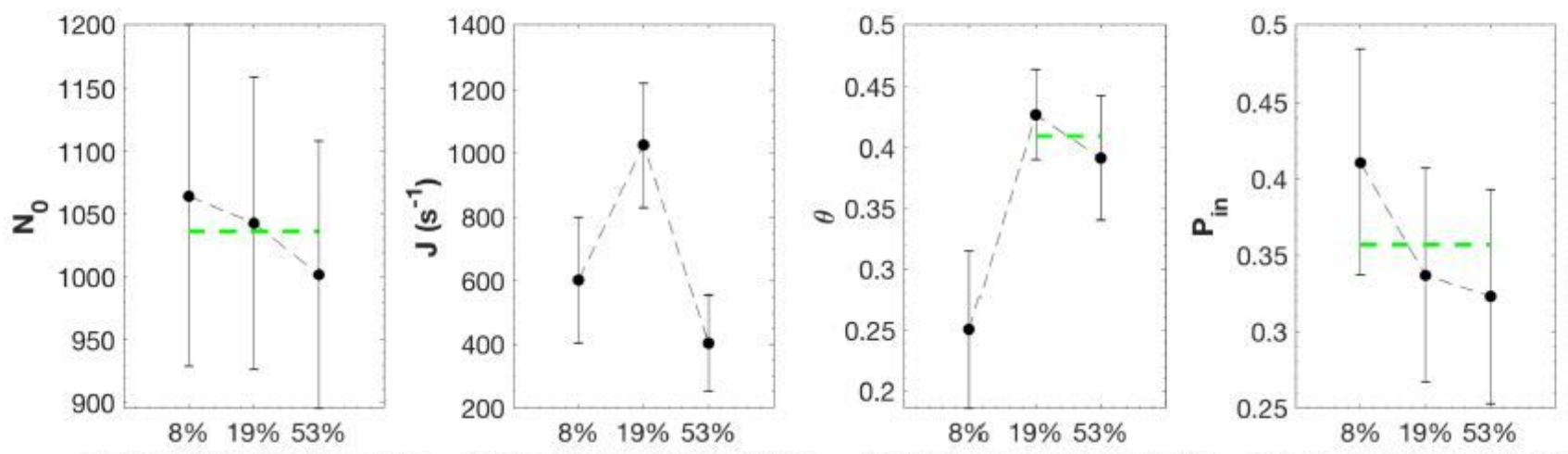

global replicated fraction global replicated fraction

global replicated fraction

global replicated fraction
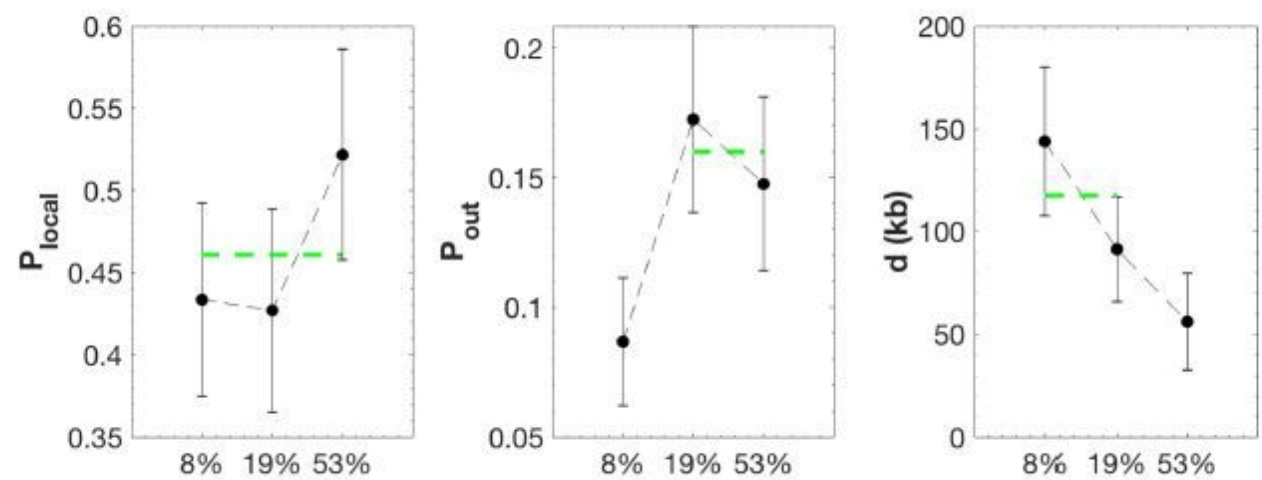

global replicated fraction

global replicated fraction

global replicated fraction

\section{Figure 4}

"Please see the Manuscript PDF file for the complete figure caption". 
a

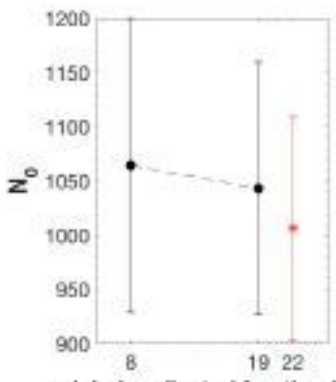

global replicated fraction $(\%)$

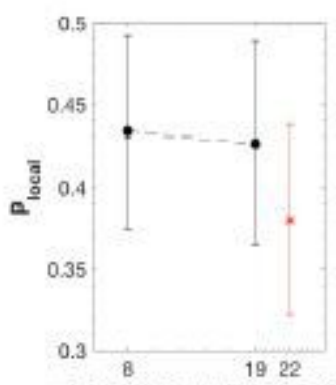

alobal reblicated fraction $(\%)$
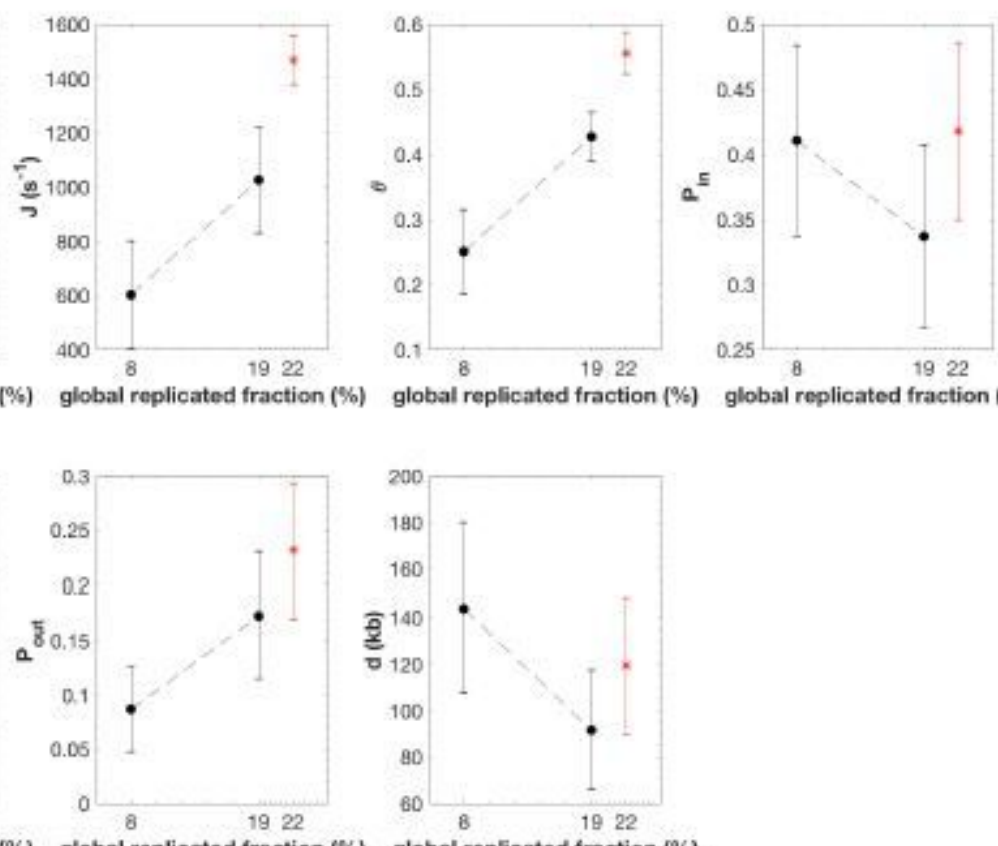

global replicated fraction (\%)

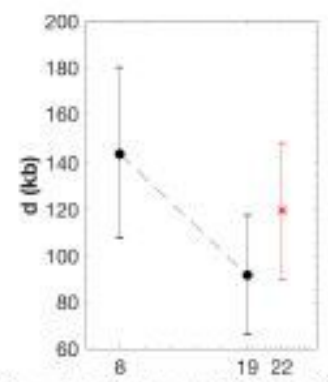

b
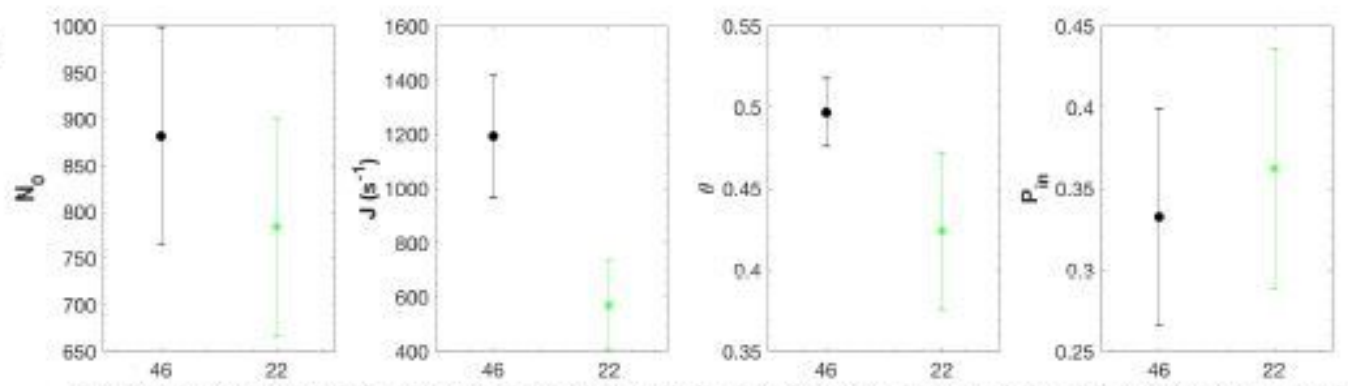

global replicated fraction (\%) global replicated fraction $(\%)$
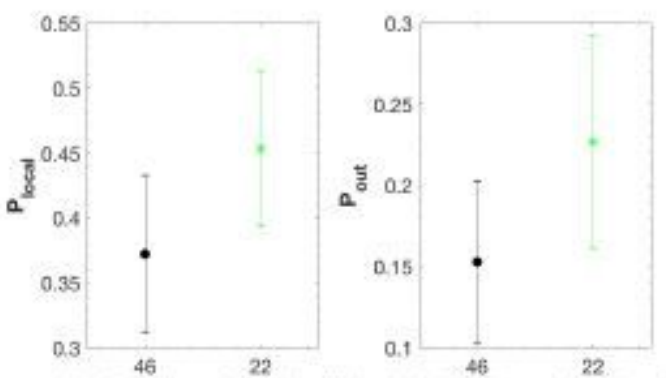

global replicated fraction (\%) global replicated fraction $(\%)$

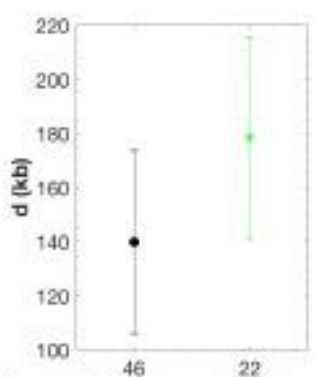

global replicated fraction $(\%)$

\section{Figure 5}

"Please see the Manuscript PDF file for the complete figure caption". 
a
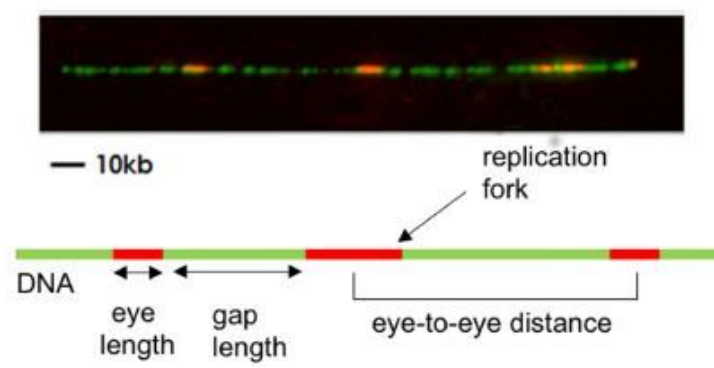

b

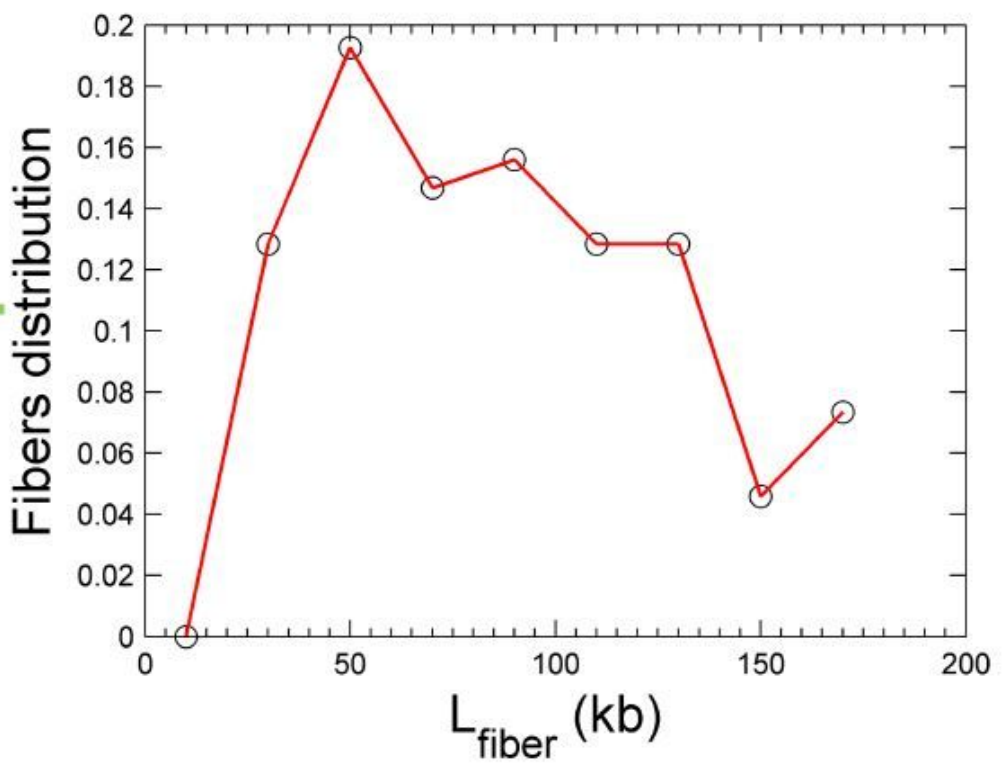

Figure 6

"Please see the Manuscript PDF file for the complete figure caption".

\section{Supplementary Files}

This is a list of supplementary files associated with this preprint. Click to download.

- S1.pdf

- S2.pdf

- S3.pdf 